\title{
Prokaryotic Expression of Phosphoenolpyruvate Carboxylase Fragments from Peanut and Analysis of Osmotic Stress Tolerance of Recombinant Strains
}

\author{
Jiaqi Tu ${ }^{1}$, Lanlan Feng ${ }^{1}$, Yanbin Hong ${ }^{2}$, Qiuyun Liu ${ }^{1}$, Xia Huang ${ }^{1}$ and Yin $_{\text {Li }}{ }^{1} * *$ \\ 1 Guangdong Key Laboratory of Plant Resources, School of Life Sciences, Sun Yat-sen University, \\ Guangzhou 510275, China; cathytu@cyagen.com (J.T.); fengll@link.cuhk.edu.hk (L.F.); \\ lsslqy@mail.sysu.edu.cn (Q.L.); huangxia@mail.sysu.edu.cn (X.H.) \\ 2 Crops Research Institute, Guangdong Academy of Agricultural Sciences, Guangzhou 510640, China; \\ hongyanbin@gdaas.cn \\ * Correspondence: liyin@mail.sysu.edu.cn
}

check for updates

Citation: Tu, J.; Feng, L.; Hong, Y.; Liu, Q.; Huang, X.; Li, Y. Prokaryotic Expression of Phosphoenolpyruvate Carboxylase Fragments from Peanut and Analysis of Osmotic Stress Tolerance of Recombinant Strains. Plants 2021, 10, 365. https://doi.org/ $10.3390 /$ plants10020365

Academic Editors: Naoki Yamamoto and Toshio Sugimoto

Received: 29 December 2020

Accepted: 8 February 2021

Published: 14 February 2021

Publisher's Note: MDPI stays neutral with regard to jurisdictional claims in published maps and institutional affiliations.

Copyright: (c) 2021 by the authors. Licensee MDPI, Basel, Switzerland. This article is an open access article distributed under the terms and conditions of the Creative Commons Attribution (CC BY) license (https:// creativecommons.org/licenses/by/ $4.0 /)$.

\begin{abstract}
Phosphoenolpyruvate carboxylase (PEPC) is a ubiquitous cytosolic enzyme that catalyzes the irreversible $\beta$-carboxylation of phosphoenolpyruvate (PEP) in presence of $\mathrm{HCO}_{3}{ }^{-}$to produce oxaloacetate (OAA) during carbon fixation and photosynthesis. It is well accepted that PEPC genes are expressed in plants upon stress. PEPC also supports the biosynthesis of biocompatible osmolytes in many plant species under osmotic stress. There are five isoforms of $P E P C$ found in peanut (Arachis hypogaea L.), namely, AhPEPC1, AhPEPC2, AhPEPC3, AhPEPC4, and AhPEPC5. Quantitative real-time polymerase chain reaction (qRT-PCR) analysis revealed that the gene expression patterns of these $A h P E P C$ genes were different in mature seeds, stems, roots, flowers, and leaves. The expression of all the plant type PEPC (PTPCs) (AhPEPC1, AhPEPC2, AhPEPC3, and AhPEPC4) was relatively high in roots, while the bacterial type PEPC (BTPC) (AhPEPC5) showed a remarkable expression level in flowers. Principal component analysis (PCA) result showed that AhPEPC 3 and AhPEPC4 are correlated with each other, indicating comparatively associations with roots, and AhPEPC5 have a very close relationship with flowers. In order to investigate the function of these AhPEPCs, the fragments of these five AhPEPC cDNA were cloned and expressed in Escherichia coli (E. coli). The recombinant proteins contained a conserved domain with a histidine site, which is important for enzyme catalysis. Results showed that protein fragments of AhPEPC1, AhPEPC2, and AhPEPC5 had remarkable expression levels in $E$. coli. These three recombinant strains were more sensitive at $\mathrm{pH} 9.0$, and recombinant strains carrying AhPEPC2 and AhPEPC5 fragments exhibited more growth than the control strain with the presence of PEG6000. Our findings showed that the expression of the $A$ PEPC fragments may enhance the resistance of transformed E. coli to osmotic stress.
\end{abstract}

Keywords: phosphoenolpyruvate carboxylase; Arachis hypogaea L.; Escherichia coli; recombinant; osmotic stress

\section{Introduction}

Phosphoenolpyruvate carboxylase (PEPC; EC 4.1.1.31) is a key enzyme in plant metabolic pathways such as photosynthesis. PEPC is a tightly regulated cytosolic enzyme in cytoplasm, catalyzing the irreversible $\beta$-carboxylation of PEP in presence of $\mathrm{HCO}_{3}{ }^{-}$ to produce oxaloacetate (OAA) and inorganic phosphorous (Pi) with magnesium $\left(\mathrm{Mg}^{2+}\right)$ ions as a cofactor [1,2]. PEPC is abundantly present in the plant kingdom as well as green algae and cyanobacteria, most of the archaea, and non-photosynthetic bacteria [2]. On the basis of function and phylogeny, the PEPCs can be divided into two subfamilies, the plant type PEPC (PTPC) and the bacterial type PEPC (BTPC). PTPC genes encode proteins of size 100-110 kDa with a conserved N-terminal seryl-phosphorylation site and a critical C terminal tetrapeptide QNTG. It is reported that all PTPCs originated from a common ancestor [3-6]. BTPC genes encode larger proteins (116-118 kDa) of partial similarity with 
PTPC, which has a prokaryotic-like (R/K) NTG C-terminal tetrapeptide $[5,6]$. Both PTPCs and BTPCs have the important domains required for catalytic and substrate binding, the BTPCs resemble the bacterial PEPCs rather than the common plant PEPCs. BTPC proteins do not possess N-terminal seryl-phosphorylation domain of PTPCs $[7,8]$.

The role and regulation for PEPCs in plants have been extensively investigated due to their important role in carbon fixation and photosynthesis [1,2]. PEPCs in plants are involved in the synthesis of storage compounds by the anaplerotic reaction. These storage compounds enter the tricarboxylic acid cycle (TCA) with intermediates such as pyruvate and phosphoenolpyruvate (PEP) involve in a variety of biosynthetic pathways [1,9]. In addition to the photosynthesis, PEPC also plays important role in carbon-nitrogen interactions, maintaining cellular $\mathrm{pH}$, seed formation and germination, fruit maturation, control of stomatal movements, supplying carbon for symbiotic $\mathrm{N}_{2}$-fixation in root nodules of leguminous plants, and regulates the mechanism of stress tolerance in plants [10-13].

Peanut (Arachis hypogaea L.) is an economically important oilseed legume distributed across more than 115 countries with 26 million hectares of area [14]. Previously, five PEPC genes (AhPEPC1, AhPEPC2, AhPEPC3, AhPEPC4, and AhPEPC5) were isolated from peanut and the complexity of the peanut $P E P C$ gene family was analyzed using phylogenetic relationship, gene structure, and chromosome mapping in peanut $[15,16]$. It was reported that AhPEPC1, AhPEPC2, AhPEPC3, and AhPEPC4 are typical PTPCs, and AhPEPC5 is a BTPC $[13,15,16]$. The interesting finding is that the expression level of AhPEPCs is lower in the high oil yielding cultivated peanut varieties than in the cultivated peanut varieties with normal oil yield except AhPEPC2 [15].

The AhPEPCs' gene expressions vary depending on the tissue, organ, and stage of development $[13,16]$. Pan et al. [13] analyzed the expression patterns of AhPEPC1-5 in peanut cultivar E11 under abiotic stress (cold, salt, and drought) conditions and observed that the expression of $A h P E P C 3$ showed high expression under abiotic stress, whereas the expression of AhPEPC1 and AhPEPC2 showed moderate expression. AhPEPC1, AhPEPC4, and AhPEPC5 were down-regulated under salt stress, and AhPEPC4 and AhPEPC5 were down-regulated under drought stress, indicating that peanut PEPCs might be playing different roles upon various abiotic stresses. However, there are few reports on the function of these five genes using the ectopic expression. Therefore, in this study, five AhPEPC coding sequences from cultivated peanut were analyzed, and cDNA segments were cloned in Escherichia coli. These AhPEPC coding sequences containing one of the five conserved domains with a histidine site were transformed into E. coli for prokaryotic expression analysis. The recombinant E. coli growth analysis showed that AhPEPC1, AhPEPC2, and AhPEPC5 had brought resistance of transformed E. coli to osmotic stress, but the recombinant strains turned out to be more sensitive to basic condition. These results provide molecular evidence for the function of the AhPEPC genes in response to stress tolerance in plants.

\section{Results}

\subsection{Expression Patterns of AhPEPCs in Peanut}

Quantitative real-time PCR (qRT-PCR) was used to monitor the expression patterns of the five $A h P E P C$ genes in different peanut tissues. The relative expression levels of $A h P E P C$ s are shown in Figure 1. The results showed that the expression of AhPEPC1 was relatively high in flowers, roots, and leaves when compared with mature seeds and stems. The expression levels of $A h P E P C 2$ in roots, flowers, leaves, and stems were relatively high, but low in seeds. The AhPEPC 3 was relatively high in roots and flowers, lower in stems, leaves, and seeds, while AhPEPC4 showed much higher accumulation in roots and flowers than in leaves, stems, and seeds. The AhPEPC 5 showed high expression in flowers, moderate in leaves, and low expression in other tissues, especially in seeds. The expression of all the PTPCs (AhPEPC1, AhPEPC2, AhPEPC3, and AhPEPC4) was relatively high in roots, while the BTPC (AhPEPC5) showed a remarkable expression level in flowers. 

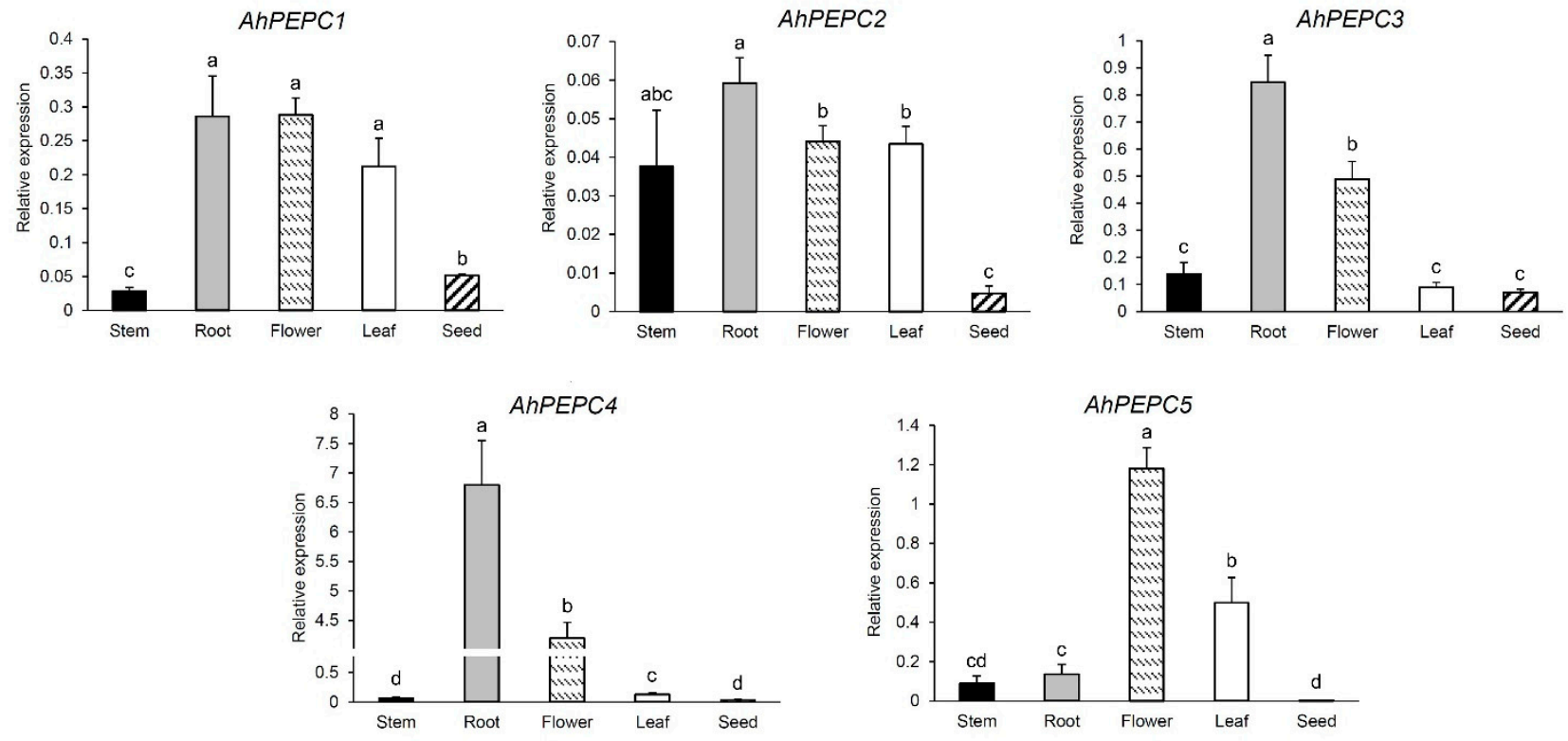

Figure 1. Expression patterns of five AhPEPC genes using qRT-PCR in different organs of peanut cultivar Shanyou 523. The graphs illustrate means \pm SD of three replicates. The bars not sharing the same letter have a statistically significant difference in gene expression level $(p<0.05)$.

The five PEPC genes exhibited different gene expression patterns, suggesting that these genes may have different roles in peanut. In order to find interrelationships among AhPEPCs gene expression levels in different organs tested, a principal component analysis (PCA) was performed (Figure 2). The two principal components PC1 and PC2 covered approximately $88.3 \%$ cumulative proportion of the total variance. Based on the distribution of different samples in the PCA biplot and contributions of variables, AhPEPC3 and $A h P E P C 4$ are correlated with each other, indicating comparative associations with roots; moreover, there is a very close relationship between AhPEPC 5 and flowers.

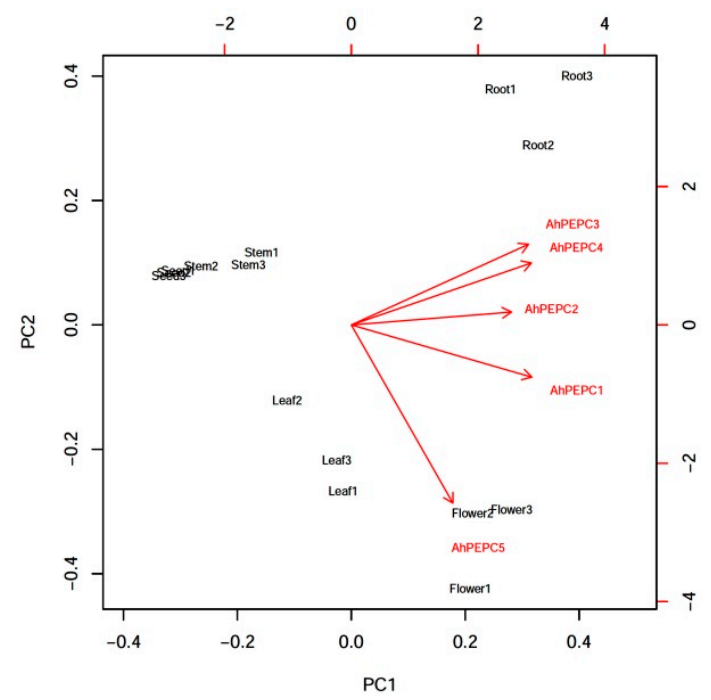

Figure 2. The results of principal component analysis (PCA) of the expression levels of the five phosphoenolpyruvate carboxylase (PEPC) genes in different peanut organs. A distribution of the samples analyzed showed by a biplot, horizontal-axes represent principal component 1 (PC1) and vertical-axes represent principal component 2 (PC2). Arrows indicate the directions of AhPEPCs gene expression levels. PC1 and PC2 explain $68.7 \%$ and $19.6 \%$ of the data variances, respectively. 


\subsection{Cloning of AhPEPC cDNA Fragments and Construction of Prokaryotic Expression Vectors}

The cDNA fragments of AhPEPC1 (688 bp), AhPEPC2 (574 bp), AhPEPC3 (538 bp), AhPEPC4 (529 bp), and AhPEPC5 (568 bp) were amplified by PCR (Supplementary Figure S1) based on the cDNA sequences of AhPEPC1 (EU391629), AhPEPC2 (FJ222240), AhPEPC3 (FJ222826), AhPEPC4 (FJ222827), and AhPEPC5 (FJ222828) from NCBI database. The fragments encoded peptides contained one of the important conserved domains (VLTAHPT) necessary for enzyme activity, harboring the catalytic site of a histidine residue [3] (Figure 3). The five AhPEPC fragments obtained are consistent with predictions, which encodes $24.64,21.01,19.14,18.89,20.24 \mathrm{kDa}$ fraction of AhPEPC1-5 protein, respectively. The five fragments were inserted into the prokaryotic expression vector $\mathrm{pET}$-28a with a $6 \times$ His tag, respectively, and then transformed into E. coli DH5 $\alpha$ and confirmed by PCR (Supplementary Materials Figure S2) and DNA sequencing. The corresponding DNA fragments were detected in agarose gel electrophoresis, and the cloned fragments are identical to the sequences in NCBI database.

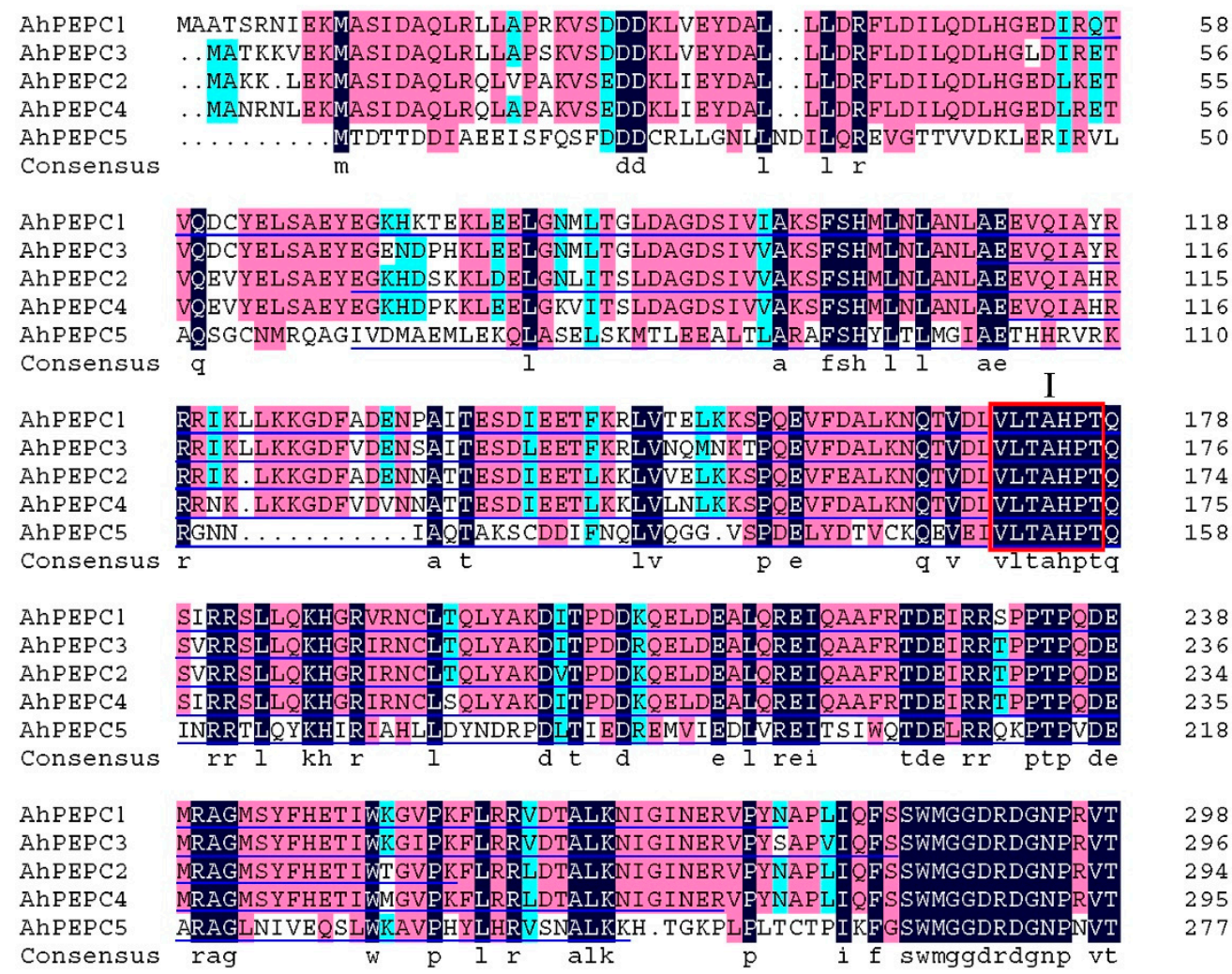

Figure 3. Alignment of part of five AhPEPC protein sequences from peanut. The underlines indicate the $A$ PEPC peptides encoded by the cloned cDNA fragments used for recombinant expression, and the box I domain is one of the conserved domains important for enzyme catalysis [3].

\subsection{Expression of AhPEPC Fragments in E. coli}

The recombinant expression vectors carrying $A$ PEEPC fragments were transformed into Rossetta (DE3) competent cells to optimize the expression conditions for the efficient production of recombinant proteins. The SDS-PAGE analysis results (Figure 4) showed that three recombinant strains with transgenes for the fragment of AhPEPCs produced the peptides of the expected size (25-33 kDa) with remarkable efficiency. The size of predicted peptides composed of $6 \mathrm{xHis}$ tag fused to the fragment of AhPEPC protein was $30.03 \mathrm{kDa}$ for AhPEPC1, AhPEPC1, $26.40 \mathrm{kDa}$ for AhPEPC2, and $25.63 \mathrm{kDa}$ for AhPEPC5. The amounts of isopropyl $\beta$-d-thiogalactopyranoside (IPTG)-induced peptides were gradually increased with increasing time of induction. 

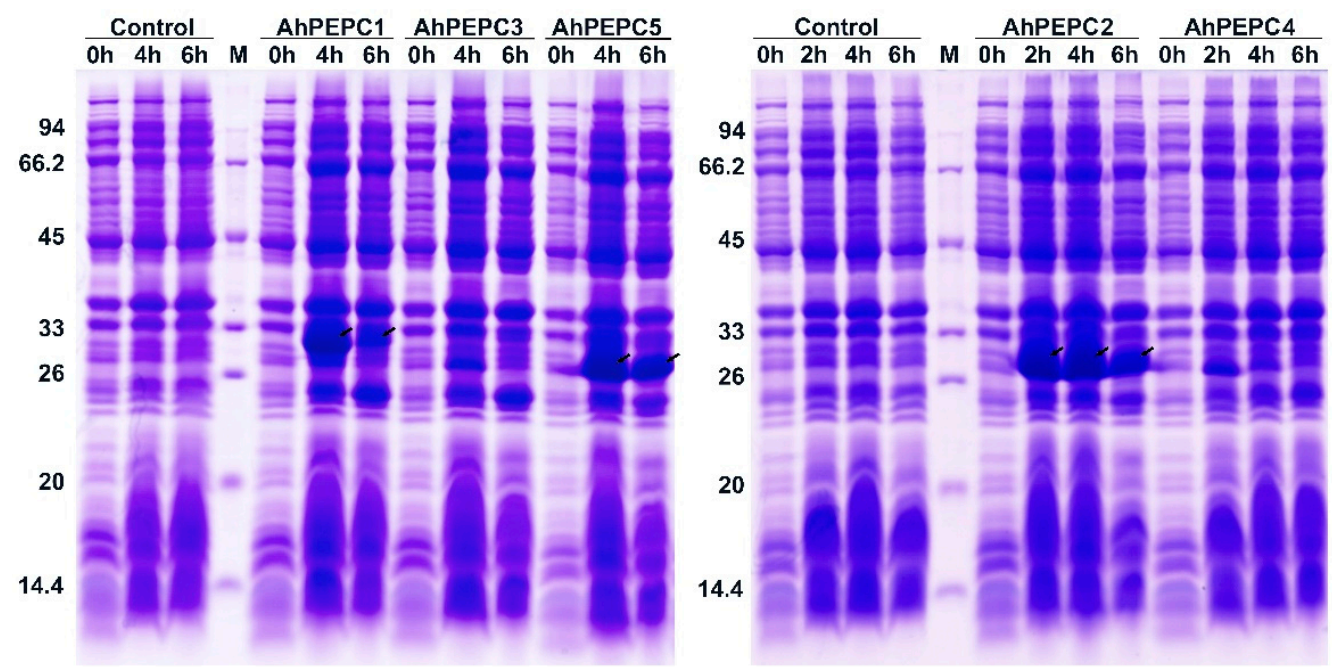

Figure 4. SDS-PAGE analysis of recombinant AhPEPC peptides expression in Escherichia coli Rossetta (DE3) with Coomassie blue staining. The pET-28a harboring stain was used as vector control. Arrows indicate the band of AhPEPC His-tag fusion peptides.

Total bacterial proteins of the three strains were tested by the SDS-PAGE and Western blot to study whether the strains contain His-tag (Figure 5). We observed that the protein band of the size of the target protein increased significantly after induction. Western blotting results showed that the recombinant peptides of AhPEPC1, AhPEPC2, and AhPEPC5 contained His-tag, and the immunoblotting of His-tag was not observed in the control.

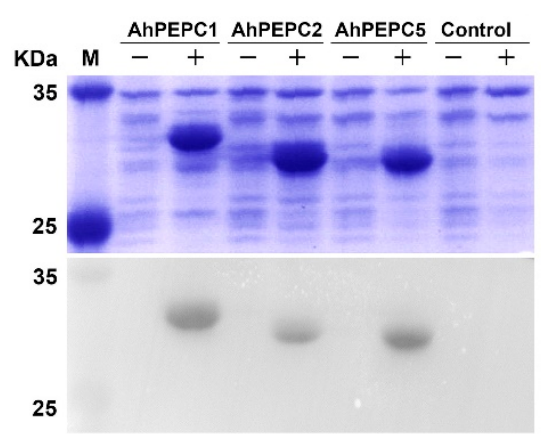

Figure 5. Western blot analysis of the expression of the recombinant AhPEPC with anti-His antibody. Specific protein bands were detected in three induced recombinant pET-28a-AhPEPC strains. M: Prestained protein molecular weight marker; +: Induced with $0.4 \mathrm{mM}$ isopropyl $\beta$ d-thiogalactopyranoside (IPTG); - : Control.

\subsection{Effect of Osmotic Stress on the Growth of Recombinant Strains}

To do a quick assessment of the peanut PEPC function, ectopic expression of AhPEPC peptides in $E$. coli were determined to evaluate whether the peptides affect the growth of $E$. coli and represent a function against osmotic stress $[17,18]$. In controlled trials, there was no apparent difference in cell growth between the AhPEPC fragments carrying strains and the control strains (Supplementary Materials Figure S3). The effects of AhPEPC1, AhPEPC2, and AhPEPC5 recombinant peptides were checked by polyethylene glycol (PEG6000) treatment. As shown in Figure 5, after $6 \mathrm{~h}$ of IPTG induction, there was no significant difference in cell growth between the AhPEPC2, AhPEPC5-expressing strains, and the vector control. However, the $A h P E P C 1$-expressing strains that produced a larger protein showed growth inhibition when compared with control (Figure 6a). The treatment of 10\% PEG6000 inhibited the growth of all recombinant strains and the vector control strains, however, AhPEPC2 expressing strains showed more tolerance to osmotic stress than other strains (Figure 6b). 


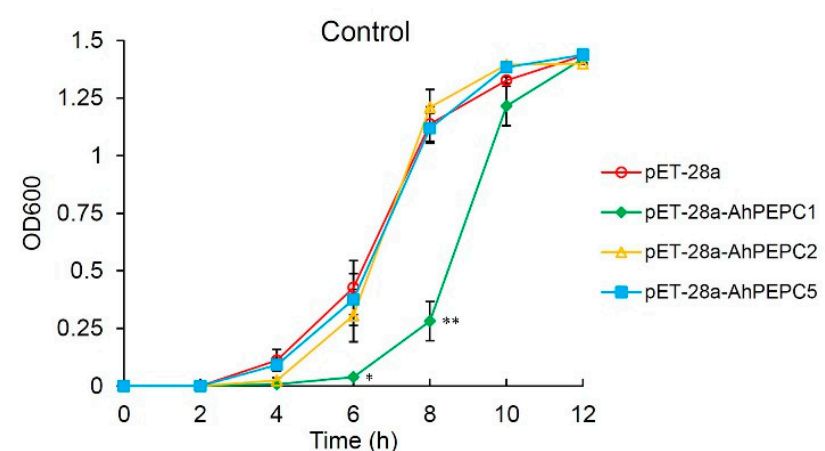

(a)

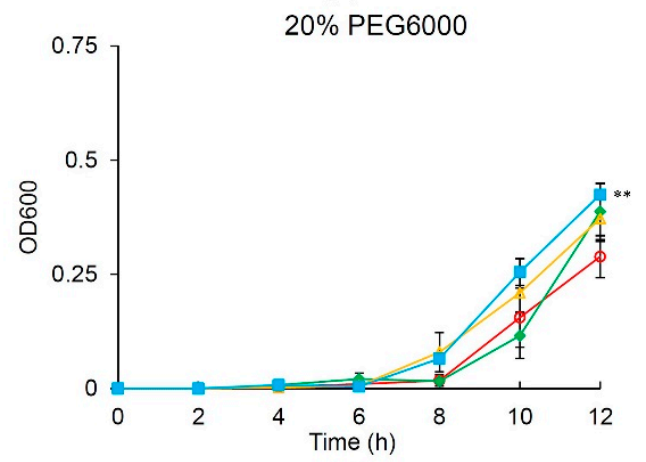

(c)



(b)

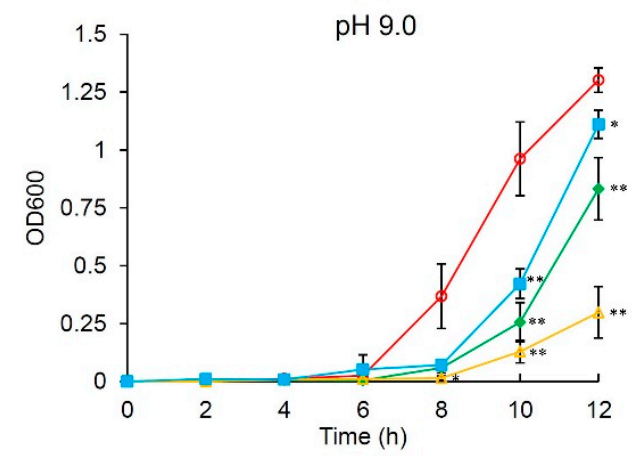

(d)

Figure 6. Time course of growth of the recombinant and control E. coli strains and the control strain under various abiotic stresses. (a) Culture under non-stressed condition after 6 h IPTG induction; (b) 10\% PEG6000; (c) 20\% PEG6000; (d) pH 9.0. The cultures were sampled at every $2 \mathrm{~h}$ interval for $12 \mathrm{~h}$. Data points represent the means $\pm \mathrm{SD}$ of three replicates. ${ }^{*} p<0.05$, ** $p<0.01$.

When the concentration of PEG6000 reached 20\%, as up to $12 \mathrm{~h}$ the AhPEPC2 expressing strains showed more tolerance, and the growth of AhPEPC1-expressing strains were inhibited to a lesser extent compared to the vector control (Figure 6c). These results showed that recombinant strains overexpressing AhPEPCs improved osmotic tolerance.

\subsection{Acid/base Tolerance Test of Recombinant Strains}

AhPEPC1, AhPEPC2, and AhPEPC5-expressing strains were cultured in media with $\mathrm{pH}$ range 3.0-9.0. In culture media of $\mathrm{pH} 3.0$ to $\mathrm{pH} 7.0$, the strains showed similar growth patterns to the vector control (data not shown). However, when the $\mathrm{pH}$ was increased to $\mathrm{pH}$ 9.0, the growth of these three recombinant strains was severely inhibited, which indicated that recombinant strains are sensitive at basic $\mathrm{pH}$ (Figure 6d).

\section{Discussion}

Plant PEPC is a key enzyme in photosynthesis, seed development [19-23], nutrient accumulation and metabolism [16,24,25], and abiotic stress adaptation [1,26-29]. One of the important seed crops, peanut, has five PEPC genes that can regulate the metabolic process of fatty acid and protein biosynthesis in seeds [16]. In the present study, we further characterized these peanut PEPCs using the prokaryotic expression technique. The results offered experimental evidence for the relation of $P E P C$ in peanut to osmotic stress.

Previous studies $[13,15]$ reported gene expression patterns of the five PEPC in roots, stems, seeds, and leaves while no report in flowers was available. In our qRT-PCR assays, we found the distinctive expression of the BTPC AhPEPC 5 in flowers. The expression patterns of the five AhPEPC genes are strikingly different between normal and high-oil varieties but do not offer any obvious clues to the function of the AhPEPC genes $[13,15]$. However, it was presumed that lower-level expression of AhPEPCs facilitates the flow of PEP into lipid in high-oil peanut variety [15]. The analysis of results during seed development (10-60 d after pegging) also presumed that lower-level expression of AhPEPC 
genes and the decrease in PEPC activity may be in favor of lipid accumulation in peanut seeds [16]. In the present study, we analyzed the AhPEPCs expression in peanut cultivar Shanyou 523, which is a normal-oil variety, and the results showed differential gene expression patterns. The plant materials used in this research were not from the same developmental stage as those in previous studies. The PCA results supported that AhPEPC4 might have an important function in roots, and the correlation of AhPEPC4 and AhPEPC 3 implies that they may have similar functions. The expression patterns of five PEPC in flowers of cultivated peanut have not been investigated before. It is notable that all five PEPCs were more abundant in flowers than in leaves. The AhPEPC5 showed a different expression pattern as it expressed much higher in flowers when compared to other organs tested. There is a remarkable connection between AhPEPC5 and flowers in the PCA biplot, indicating that this bacterial type AhPEPC may also play an important role in flower development.

Prokaryotic expression by using E. coli as a host is widely used to validate the protein function. Overexpression of plant-derived PEPC gene in E. coli can enhance tolerance when exposed to high temperature, salinity, dehydration, or methyl viologen [17], and recombinant expression of PEPC gene fragment can enhance the regulation of protein and lipid accumulation [30], suggesting that plant-derived PEPC may have a function in E. coli. In the present study, we had expressed fragments of five AhPEPCs containing one conserved domain (VLTAHPT) with an histidine site, not include other highly conserved motifs which found in plant type of PEPC [3,31]. A previous study revealed that the PEPC activity of recombinant $E$. coli harboring a peptide from Chlamydomonas reinhardtii has elevated $24 \%$ higher than wild type [30]. The protein fragments in the present study correspond to similar region of the peptide. In PEPC, there are only two conserved histidines, and site-directed mutagenesis of these histidines has identified their essential role in catalytic activity [32,33]. These two conserved histidines together with other residues are important for catalytic activity and the inhibitory effector aspartate [33]. We had expressed AhPEPC fragments containing one of the conserved domains VLTAHPT and the first histidine site. Site-directed mutagenesis and three-dimensional structure analysis suggested that this histidine site involves in the carboxylation of PEP with bicarbonate anion to form oxaloacetate [32,34]. In plants, PEPC converts PEP to OAA by carboxylation, subsequently malate and eventually lead to the synthesis and accumulation of osmotically active compounds and improves drought tolerance in plants $[18,35,36]$. Pan et al. [13] have found that PEPC genes in peanuts showed differential transcription abundance in leaves during cold, salt, and drought stresses, suggesting functions of regulation in responses to abiotic stresses. Our investigations did not find the recombinant strains showing resistance to high or low temperature, salinity, and oxidative stress (data not shown), These incomplete peptides could not have full activity but may compete for a substrate with native enzymes, and the ectopic expression of AhPEPC2 and AhPEPC5 fragments may have conferred osmotic stress resistance to the recombinant strains to a certain extent. Further investigation is needed to study whether the whole or larger fragment of the protein shows better effect.

Park et al. [37] expressed a PEPC protein from marine bacteria Oceanimonas smirnovii in E. coli and found that the purified PEPC protein maintains enzymatic activity in basic $\mathrm{pH}$ 9.0-10.0, but almost no activity at acidic $\mathrm{pH}$ 5.0. PEPC up-regulation may increase organic acid synthesis, including malate and citrate, and PEPC activities in root tips of plants are much higher at basic $\mathrm{pH}$ [38]. PEPC also participate in proton sink through the regulation of malate and pyruvate metabolism [39]. Cheng et al. [18] found that recombinant strain expressing a PEPC protein from C4 halophyte Suaeda aralocaspica could adapt to a wider range of $\mathrm{pH}$ at 5.0 and 9.0 than that of control, but we did not find similar results in this study. In the present study, the recombinant strains showed more sensitivity to basic conditions and showed growth inhibition under basic $\mathrm{pH} 9.0$. It is possible that the incomplete $A$ PEEPC peptides had led to substrate competition but no corresponding proton transfer reaction occurs, which leads to the recombinant strains being more sensitive to the basic environment. 


\section{Materials and Methods}

\subsection{Plant Materials, E. coli Strains, and Plasmids}

A peanut cultivar 'Shanyou 523' was provided by the Crop Research Institute, Guangdong Academy of Agricultural Sciences (Guangzhou, China). Seeds were planted in 2.5-L soil-containing pots and grown till the flower stage in a greenhouse, at $25{ }^{\circ} \mathrm{C}$ with a 14 $\mathrm{h}$ light and $10 \mathrm{~h}$ dark cycle. Plant material used for RNA isolation was collected from 50 days old peanut plants at full flowering stage. Root tissues were collected from the young growing part of the main roots. Leaf and stem tissues were collected from the third compound leaf below the terminal bud and the corresponding stem segment of the sampled leaves and the mature seeds were collected about 50 days after pegging. Collected samples were immediately frozen and stored at $-80^{\circ} \mathrm{C}$ until use. Three biological replicates were used for each treatment. E. coli strain DH5 $\alpha$ and Rossetta (DE3) was from Tiangen Biotech (Beijing, China) Co. Ltd. The vector $\mathrm{pET}-28 \mathrm{a}$ was procured from Beijing Dingguo Changsheng Biotechnology Co. Ltd. (Beijing, China).

\subsection{AhPEPC Fragments Cloning and Construction of Expression Vectors}

Total RNA was extracted using the RNAprep Pure Plant Kit (Polysaccharides and Polyphenolics-rich) (Tiangen, Beijing, China), cDNA was synthesized according to the instruction in PrimeScript ${ }^{\mathrm{TM}}$ 1st Strand cDNA Synthesis Kit (TaKaRa, Kyoto, Japan). Primer pairs for the five PEPCs in peanut were designed based on the cDNA sequences available in NCBI database, these were AhPEPC1 (accession number: EU391629), AhPEPC2 (accession number: FJ222240), AhPEPC3 (accession number: FJ222826), AhPEPC4 (accession number: FJ222827), and AhPEPC5 (accession number: FJ222828). DNAMAN software was used for multiple sequence alignment. The gene fragments were amplified with gene-specific primers (Table S1), an initiation codon (ATG) and an EcoR I site were incorporated in the forward primers. Gene fragments were digested with restriction enzymes and ligated into a prokaryotic expression vector (pET28a, Beijing Dingguo Changsheng Biotechnology Co. Ltd., Beijing, China) to generate the expression plasmid pET28a-AhPEPCs. PCR reaction was carried out as follows: initial denaturation $\left(94{ }^{\circ} \mathrm{C}, 2 \mathrm{~min}\right), 32$ cycles $\left(94{ }^{\circ} \mathrm{C}\right.$ for $15 \mathrm{~s}$, $58{ }^{\circ} \mathrm{C}$ for $30 \mathrm{~s}, 72{ }^{\circ} \mathrm{C}$ for $60 \mathrm{~s}$ ), and final extension of $72{ }^{\circ} \mathrm{C}$ for $10 \mathrm{~min}$. The resultant AhPEPC fragments generated from PCR were inserted into the pET-28a vector to construct the prokaryotic expression vectors. The heat shock method was used to transform the plasmids into E. coli DH5 $\alpha$. The positive clones were selected by PCR and then verified by sequencing.

\subsection{Quantitative Gene Expression Analysis}

Expression patterns of AhPEPCs in peanut tissues were investigated using qRT-PCR, and total RNA was extracted from roots, stems, leaves, and mature seeds of flowering peanut plant. Subsequently, cDNA was synthesized. Transcript-specific primers were designed and the peanut $18 S$ rRNA was taken as the reference gene to calculate the expression levels [15]. Primers were presented in Table S2. The qRT-PCR reaction mixtures were prepared using a Premix Ex TaqTM (TaKaRa, Kyoto, Japan), reactions were performed as the manufacturer's instructions on an ABI StepOne Plus system [40]. Each $20 \mu \mathrm{L}$ reaction contained $2.0 \mu \mathrm{L}$ template, $10 \mu \mathrm{L} 2 \times$ Premix Ex TaqTM, and $0.8 \mu \mathrm{L}(10 \mu \mathrm{M})$ of each primer. qRT-PCR was conducted at initial denaturation $95^{\circ} \mathrm{C}, 3 \mathrm{~min}$, then 40 cycles of $95^{\circ} \mathrm{C}, 10 \mathrm{~s}, 60{ }^{\circ} \mathrm{C}, 15 \mathrm{~s}$, and $72{ }^{\circ} \mathrm{C}, 20 \mathrm{~s}$. Relative expression was calculated using the $2^{-\Delta \Delta C T}$ method [41]. Each measurement was carried out in triplicate with three biological replicates.

\subsection{Prokaryotic Expression and Western Blot Analysis}

The prokaryotic expression strain E. coli Rossetta (DE3) was transformed with the correctly sequenced plasmid. For prokaryotic expression analysis, the strains were cultured on a rotary shaker with incubator at $37{ }^{\circ} \mathrm{C}$ and $200 \mathrm{rpm}$ speed. During log phase, an isopropyl $\beta$-d-thiogalactopyranoside (IPTG) concentration of $0.4 \mathrm{mM}$ was used to induce 
the fusion protein expression at $37^{\circ} \mathrm{C}$ for $6 \mathrm{~h}$. The bacterial suspension was harvested by centrifugation for $0,2,4$, and $6 \mathrm{~h}$, and the fusion protein was separated by $12 \%$ sodium dodecyl sulfate-polyacrylamide gel electrophoresis (SDS-PAGE) and visualized using Coomassie brilliant blue (CBB) staining. Protein samples were transferred to an Immobilon Polyvinylidene Fluoride (PVDF) membrane (Millipore, Burlington, MA, USA), 5\% skim milk powder was used to block the membrane. Membranes were treated with His-Tag mouse monoclonal antibody (Proteintech) and a secondary antibody of horseradish peroxidase (HRP) conjugated affinipure goat anti-mouse $\operatorname{IgG}(\mathrm{H}+\mathrm{L})$ (Proteintech), and detected using Chemiluminescence Ecl Detection Kit (Millipore, Burlington, MA, USA).

\subsection{Growth and Stress Tolerance Assay}

The strains Rossetta: pET-28a-AhPEPCs (recombinant) and Rossetta: pET-28a (control) were inoculated into $5 \mathrm{~mL}$ fresh Luria-Bertani (LB) medium and incubated at $37^{\circ} \mathrm{C}$ overnight. Cultures were diluted at proportion of 1:100 into fresh LB and grown at $37^{\circ} \mathrm{C}$ for $2.5 \mathrm{~h}$. When the optical density at $600 \mathrm{~nm}$ (OD600) reached 0.6, IPTG was added to 0.4 $\mathrm{mM}$ to induce protein expression for $12 \mathrm{~h}$. The growth rate of recombinant and control strains was measured by recording the OD600 at $2 \mathrm{~h}$ interval. To perform stress tolerance assay, the recombinant strains and control strain were incubated; when the OD600 reached 0.6 , IPTG was added to $0.4 \mathrm{mM}$ to induce protein expression for $6 \mathrm{~h}$. The OD600 values were measured and the cultures were diluted to 0.5 OD600, then $200 \mu \mathrm{L}$ of each culture were inoculated into $20 \mathrm{~mL}$ fresh LB medium containing 10\%, 15\%, 20\% polyethylene glycol (PEG) 6000 for osmotic stress assay. For acid and base tolerance test, the $\mathrm{pH}$ value of the culture was adjusted to 3.0, 5.0, 7.0, 9.0. All cultures were incubated on a shaking incubator at $220 \mathrm{rpm}$ overnight at $37^{\circ} \mathrm{C}$. Growth rates were measured by recording the OD600 every $2 \mathrm{~h}$. Three biological repeats were carried out for each treatment.

\subsection{Statistical Analysis and PCA Analysis}

Data were analyzed using SPSS Statistics 26.0. Statistical analyses were performed by Student's $t$-test. All data were expressed as means \pm standard deviation (SD). $p<0.05$ and $p<0.01$ are considered statistically significant. A PCA analysis was conducted for the expression levels of the five PEPC genes using the R statistical package (https:/ / www.rproject.org/ accessed on 5 February 2021).

Supplementary Materials: The following are available online at https:/ /www.mdpi.com/2223-774 7/10/2/365/s1, Figure S1: Cloning of AhPEPC gene fragments; Figure S2: PCR identification of $E$. coli strains transformed with pET-28a-AhPEPCs; Figure S3: Growth of the recombinant E. coli strains and the control strain under a normal culture condition; Table S1: List of primer sequences used for cloning; Table S2: List of primer sequences used for qRT-PCR.

Author Contributions: Conceptualization, Y.L. and J.T.; methodology, Y.L., L.F., and J.T.; software, J.T. and L.F., validation, J.T., L.F., Y.L., and Y.H.; formal analysis, J.T., L.F., and Y.H.; investigation, J.T. and L.F.; resources, J.T., L.F., and Y.H.; data curation, J.T., Y.L., and Y.H.; writing-original draft preparation, J.T. and Y.L.; writing-review and editing, Y.L., X.H., and Q.L.; funding acquisition, Y.L. and X.H. All authors have read and agreed to the published version of the manuscript.

Funding: This research was funded by the National Natural Science Foundation of China (grant No. 31171625), the Guangzhou Municipal Science and Technology Project (grant No. 201510010270), and the Guangdong Natural Science Foundation (grant No. 2018A030313294).

Institutional Review Board Statement: "Not applicable" for studies not involving humans or animals.

Informed Consent Statement: "Not applicable" for studies not involving humans.

Data Availability Statement: The data presented in this study are available on request from the corresponding author.

Conflicts of Interest: The authors declare no conflict of interest. 


\section{References}

1. Chen, M.; Tang, Y.; Zhang, J.; Yang, M.; Xu, Y. RNA interference-based suppression of phosphoenolpyruvate carboxylase results in susceptibility of rapeseed to osmotic stress. J. Integr. Plant Biol. 2010, 52, 585-592. [CrossRef]

2. O' Leary, B.; Park, J.; Plaxton, W.C. The remarkable diversity of plant PEPC (phosphoenolpyruvate carboxylase): Recent insights into the physiological functions and post-translational controls of non-photosynthetic PEPCs. Biochem. J. 2011, 436, 15-34. [CrossRef]

3. Sánchez, R.; Cejudo, F.J. Identification and expression analysis of a gene encoding a bacterial-type phosphoenolpyruvate carboxylase from Arabidopsis and rice. Plant Physiol. 2003, 132, 949-957. [CrossRef]

4. Svensson, P.; Bläsing, O.E.; Westhoff, P. Evolution of C4 phosphoenolpyruvate carboxylase. Arch. Biochem. Biophys. 2003, 414, 180-188. [CrossRef]

5. Izui, K.; Matsumura, H.; Furumoto, T.; Kai, Y. Phosphoenolpyruvate carboxylase: A new era of structural biology. Annu. Rev. Plant Biol. 2004, 55, 69-84. [CrossRef] [PubMed]

6. $\quad$ O' Leary, B.; Fedosejevs, E.T.; Hill, A.T.; Bettridge, J.; Park, J.; Rao, S.K.; Leach, C.A.; Plaxton, W.C. Tissue-specific expression and post-translational modifications of plant- and bacterial-type phosphoenolpyruvate carboxylase isozymes of the castor oil plant, Ricinus communis L. J. Exp. Bot. 2011, 62, 5485-5495. [CrossRef]

7. Igawa, T.; Fujiwara, M.; Tanaka, I.; Fukao, Y.; Yanagawa, Y. Characterization of bacterial-type phosphoenolpyruvate carboxylase expressed in male gametophyte of higher plants. BMC Plant Biol. 2010, 10, 200. [CrossRef] [PubMed]

8. Waseem, M.; Ahmad, F. The phosphoenolpyruvate carboxylase gene family identification and expression analysis under abiotic and phytohormone stresses in Solanum lycopersicum L. Gene 2019, 690, 11-20. [CrossRef]

9. Miyao, M.; Fukayama, H. Metabolic consequences of overproduction of phosphoenolpyruvate carboxylase in C3 plants. Arch. Biochem. Biophys. 2003, 414, 197-203. [CrossRef]

10. Sánchez, R.; Flores, A.; Cejudo, F.J. Arabidopsis phosphoenolpyruvate carboxylase genes encode immunologically unrelated polypeptides and are differentially expressed in response to drought and salt stress. Planta 2006, 223, 901-909. [CrossRef] [PubMed]

11. Doubnerová Hysková, V.; Miedzińska, L.; Dobrá, J.; Vankova, R.; Ryšlavá, H. Phosphoenolpyruvate carboxylase, NADP-malic enzyme, and pyruvate, phosphate dikinase are involved in the acclimation of Nicotiana tabacum L. to drought stress. J. Plant Physiol. 2014, 171, 19-25. [CrossRef]

12. Wang, N.; Zhong, X.; Cong, Y.; Wang, T.; Yang, S.; Li, Y.; Gai, J. Genome-wide analysis of phosphoenolpyruvate carboxylase gene family and their response to abiotic stresses in soybean. Sci. Rep. 2016, 6, 38448. [CrossRef]

13. Pan, L.; Zhang, J.; Chen, N.; Chen, M.; Wang, M.; Wang, T.; Chi, X.; Yuan, M.; Wan, Y.; Yu, S.; et al. Molecular Characterization and Expression Profiling of the Phosphoenolpyruvate Carboxylase Genes in Peanut (Arachis hypogaea L.). J. Plant Physiol. 2017, 64, 576-587. [CrossRef]

14. Liu, H.; Hong, Y.; Lu, Q.; Li, H.; Gu, J.; Ren, L.; Deng, L.; Zhou, B.; Chen, X.; Liang, X. Integrated Analysis of Comparative Lipidomics and Proteomics Reveals the Dynamic Changes of Lipid Molecular Species in High-Oleic Acid Peanut Seed. J. Agric. Food Chem. 2020, 68, 426-438. [CrossRef] [PubMed]

15. Yu, S.; Pan, L.; Yang, Q.; Chen, M.; Zhang, H. Identification and Expression Analysis of the Phosphoenolpyruvate Carboxylase Gene Family in Peanut (Arachis hypogaea L.). Agric. Sci. 2010, 9, 477-487. [CrossRef]

16. Pan, L.; Yang, Q.; Chi, X.; Chen, M.; Yang, Z.; Chen, N.; Wang, T.; Wang, M.; He, Y.; Yu, S. Functional analysis of the phosphoenolpyruvate carboxylase on the lipid accumulation of peanut (Arachis hypogaea L.) seeds. J. Integr. Agric. 2013, 12, 36-44. [CrossRef]

17. Singh, J.; Reddy, G.M.; Agarwal, A.; Chandrasekhar, K.; Sopory, S.K.; Reddy, M.K.; Kaul, T. Molecular and structural analysis of C4-specific PEPC isoform from Pennisetum glaucum plays a role in stress adaptation. Gene 2012, 500, 224-231. [CrossRef] [PubMed]

18. Cheng, G.; Wang, L.; Lan, H. Cloning of PEPC-1 from a C4 halophyte Suaeda aralocaspica without Kranz anatomy and its recombinant enzymatic activity in responses to abiotic stresses. Enzyme Microb. Tech. 2016, 83, 57-67. [CrossRef]

19. Yamamoto, N.; Sasou, A.; Saito, Y.; Sugimoto, T.; Masumura, T. Protein and gene expression characteristics of a rice phosphoenolpyruvate carboxylase Osppc3; its unique role for seed cell maturation. J. Cereal Sci. 2015, 64, 100-108. [CrossRef]

20. Yamamoto, N.; Kubota, T.; Masumura, T.; Shiraishi, N.; Tanaka, K.; Sugimoto, T.; Oji, Y. Molecular cloning, gene expression and functional expression of a phosphoenolpyruvate carboxylase Osppc1 in developing rice seeds: Implication of involvement in nitrogen accumulation. Seed Sci. Res. 2014, 24, 23-36. [CrossRef]

21. Yamamoto, N.; Masumura, T.; Yano, K.; Sugimoto, T. Pattern analysis suggests that phosphoenolpyruvate carboxylase in maturing soybean seeds promotes the accumulation of protein. Biosci. Biotechnol. Biochem. 2019, 83, 2238-2243. [CrossRef] [PubMed]

22. Yamamoto, N.; Sugimoto, T.; Takano, T.; Sasou, A.; Morita, S.; Yano, K.; Masumura, T. The plant-type phosphoenolpyruvate carboxylase Gmppc2 is developmentally induced in immature soy seeds at the late maturation stage: A potential protein biomarker for seed chemical composition. Biosci. Biotechnol. Biochem. 2020, 84, 552-562. [CrossRef]

23. Yamamoto, N.; Takano, T.; Masumura, T.; Sasou, A.; Morita, S.; Sugimoto, T.; Yano, K. Rapidly evolving phosphoenolpyruvate carboxylase Gmppc1 and Gmppc7 are highly expressed in the external seed coat of immature soybean seeds. Gene 2020, $762,145015$. [CrossRef] 
24. Andre, C.; Froehlich, J.E.; Moll, M.R.; Benning, C. A heteromeric plastidic pyruvate kinase complex involved in seed oil biosynthesis in Arabidopsis. Plant Cell 2007, 19, 2006-2022. [CrossRef] [PubMed]

25. Junker, B.H.; Lonien, J.; Heady, L.E.; Rogers, A.; Schwender, J. Parallel determination of enzyme activities and in vivo fluxes in Brassica napus embryos grown on organic or inorganic nitrogen source. Phytochemistry 2007, 68, 2232-2242. [CrossRef]

26. González, M.C.; Sánchez, R.; Cejudo, F.J. Abiotic stresses affecting water balance induce phosphoenolpyruvate carboxylase expression in roots of wheat seedlings. Planta 2003, 216, 985-992. [CrossRef]

27. García-Mauriño, S.; Monreal, J.A.; Alvarez, R.; Vidal, J.; Echevarríaa, C. Characterization of salt stress-enhanced phosphoenolpyruvate carboxylase kinase activity in leaves of Sorghum vulgare: Independence from osmotic stress, involvement of ion toxicity and significance of dark phosphorylation. Planta 2003, 216, 648-655. [CrossRef]

28. Carmo-Silva, A.E.; Bernardes da Silva, A.; Keys, A.J.; Parry, M.A.J.; Arrabaça, M.C. The activities of PEP carboxylase and the C4 acid decarboxylases are little changed by drought stress in three C4 grasses of different subtypes. Photosynth. Res. 2008, 97, 223-233. [CrossRef] [PubMed]

29. Qin, N.; Xu, W.; Hu, L.; Wang, H.; Qi, X.; Fang, Y.; Hua, X. Drought tolerance and proteomics studies of transgenic wheat containing the maize C4 phosphoenolpyruvate carboxylase (PEPC) gene. Protoplasma 2016, 253, 1503-1512. [CrossRef] [PubMed]

30. Tian, Q.; Shi, D.; Jia, X.; Mi, H.; Huang, X.; He, P. Recombinant expression and functional analysis of a Chlamydomonas reinhardtii bacterial-type phosphoenolpyruvate carboxylase gene fragment. Biotechnol. Lett. 2014, 36, 821-827. [CrossRef] [PubMed]

31. Lepiniec, L.; Vidal, J.; Chollet, R.; Gadal, P.; Crétin, C. Phosphoenolpyruvate carboxylase: Structure, regulation and evolution. Plant Sci. 1994, 99, 111-124. [CrossRef]

32. Terada, K.; Izui, K. Site-directed mutagenesis of the conserved histidine residue of phosphoenolpyruvate carboxylase. European Journal of Biochemistry. Eur. J. Biochem. 1991, 202, 797-803. [CrossRef] [PubMed]

33. Kai, Y.; Matsumura, H.; Inoue, T.; Terada, T.; Nagara, Y.; Yoshinaga, T.; Kihara, A.; Tsumura, K.; Izui, K. Three-dimensional structure of phosphoenolpyruvate carboxylase: A proposed mechanism for allosteric inhibition. Proc. Natl. Acad. Sci. USA 1999, 96, 823-828. [CrossRef] [PubMed]

34. Kai, Y.; Matsumura, H.; Izui, K. Phosphoenolpyruvate carboxylase: Three-dimensional structure and molecular mechanisms. Arch. Biochem. Biophys. 2003, 414, 170-179. [CrossRef]

35. Seki, M.; Umezawa, T.; Urano, K.; Shinozaki, K. Regulatory metabolic networks in drought stress responses. Curr. Opin. Plant Biol. 2007, 10, 296-302. [CrossRef]

36. Doubnerová, V.; Ryšlavá, H. What can enzymes of C4 photosynthesis do for C3 plants under stress? Plant Sci. 2011, 180, 575-583. [CrossRef]

37. Park, S.; Lee, W.; Kim, H.; Pack, S.P.; Lee, J. Characterization of Phosphoenolpyruvate Carboxylase from Oceanimonas smirnovii in Escherichia coli. Appl. Biochem. Biotechnol. 2015, 177, 217-225. [CrossRef]

38. López-Millán, A.F.; Morales, F.; Andaluz, S.; Gogorcena, Y.; Abadía, A.; De Las Rivas, J.; Abadía, J. Responses of sugar beet roots to iron deficiency. Changes in carbon assimilation and oxygen use. Plant Physiol. 2000, 124, 885-898. [CrossRef]

39. Sakano, K. Revision of biochemical pH-stat: Involvement of alternative pathway metabolisms. Plant. Cell Physiol. 1998, 39, 467-473. [CrossRef]

40. Wen, S.; Liu, H.; Li, X.; Chen, X.; Hong, Y.; Li, H.; Lu, Q.; Liang, X. TALEN-mediated targeted mutagenesis of fatty acid desaturase 2 (FAD2) in peanut (Arachis hypogaea L.) promotes the accumulation of oleic acid. Plant Mol. Biol. 2018, 97, 177-185. [CrossRef] [PubMed]

41. Livak, K.J.; Schmittgen, T.D. Analysis of relative gene expression data using real-time quantitative PCR and the $2^{-\Delta \Delta C T}$ method. Methods 2001, 25, 402-408. [CrossRef] [PubMed] 